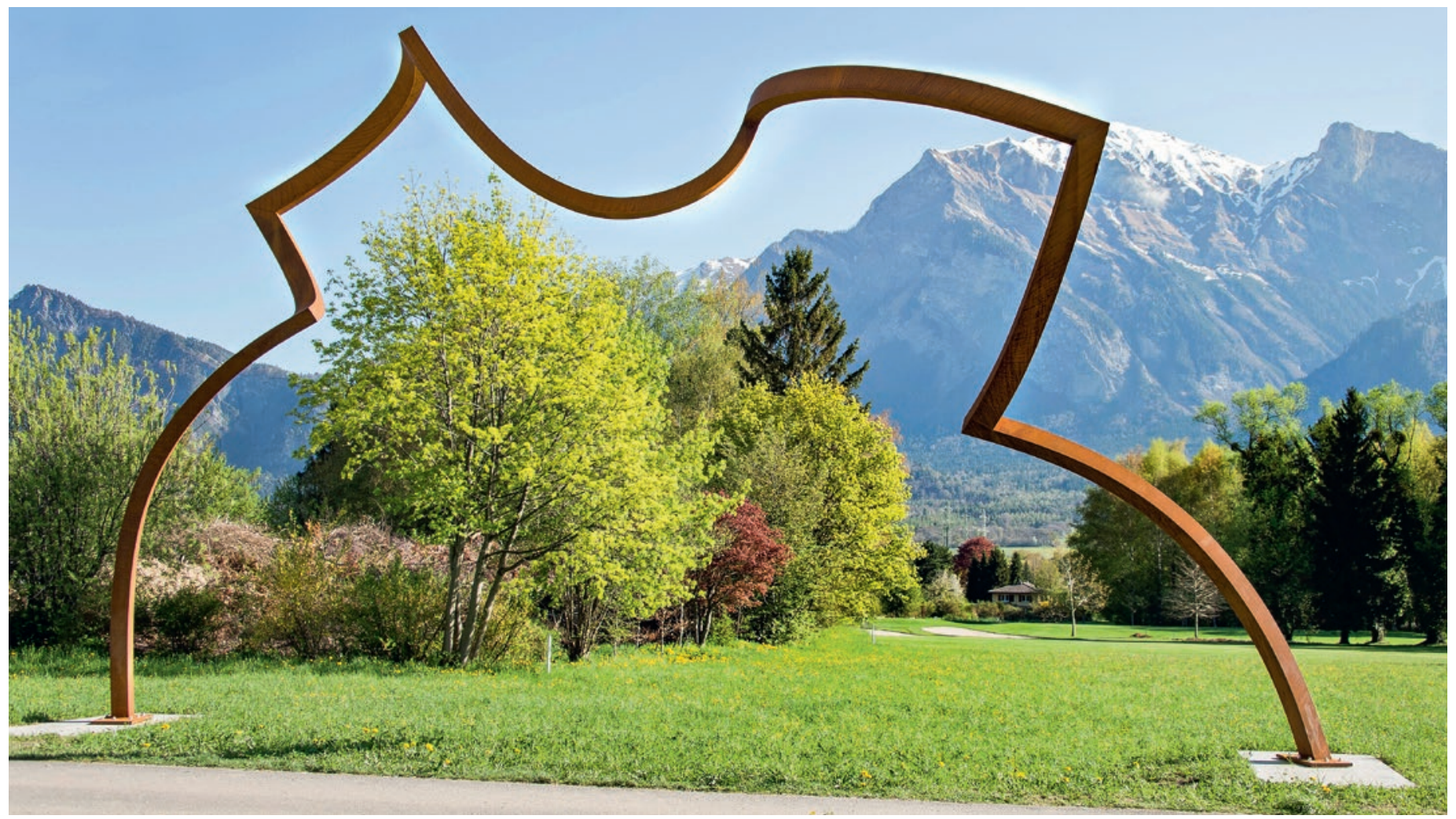

\title{
Kunst als Lebensnerv und Zeichen der Hoffnung
}

\section{Andrin Schütz}

Freischaffender Journalist

Vom 5. Mai bis zum 31. Oktober findet in Bad Ragaz die 8. Schweizerische Triennale der Skulptur «Bad RagARTz» statt. Über 400 Werke von 83 verschiedenen Kunstschaffenden warten auf die Besucherinnen und Besucher. Organisiert wird der monumentale Anlass, der alle drei Jahre rund 500000 Menschen in die St. Galler Kurgemeinde lockt, vom kunstbegeisterten Arzt Dr. Rolf Hohmeister und seiner Frau Esther Hohmeister.

Die letzten Monate waren für viele von uns mehr als herausfordernd: Aus medizinischer, aber auch aus persönlicher und gesellschaftlicher Sicht. Die Pandemie hat die menschliche Gemeinschaft weltweit in ihren grundlegenden Strukturen erschüttert. Vieles, was uns in unserem Alltag stabil, vital und seelisch gesund erhält, war nicht mehr möglich und ist teilweise noch immer nicht möglich. Der Satz, «Distanz schärft den Blick», welchen wir lange vor dem globalen Aufkommen von Covid-19 als Leitmotiv für die Schweizerische Triennale der Skulptur «Bad RagARTz» bestimmt hat- ten, hat plötzlich eine ungeahnte medizinische und gesellschaftliche Tragweite erhalten. Dennoch müssen und wollen wir nach vorne schauen.

\section{Kunst schafft ein positives Umfeld}

Gerade die Kunst kann in diesen schwierigen Zeiten wesentliche Signale geben und ein positives Umfeld schaffen. Mit der «Bad RagARTz» wird ein solches, wirkmächtiges Zeichen gesetzt: 83 renommierte Künstlerinnen und Künstler aus der Schweiz und aus aller 
Welt stellen in diesem Sommer in den weitläufigen Parkanlagen von Bad Ragaz ihre Werke aus und erschaffen somit eine faszinierende Stadt aus Formen und Farben, die für alle Interessierten frei zugänglich ist. Hat sich die Triennale in den letzten 20 Jahren zur grössten Skulpturenausstellung Europas entwickelt, gibt sie nicht nur dem Tourismus und der Wirtschaft in der Region unverzichtbare und nachhaltige Impulse. Vielmehr ist sie auch zu einem weit über die Region hinaus wichtigen gesellschaftlichen Faktor geworden: Abertausende von Menschen geniessen alle drei Jahre entspannende und anregende Spaziergänge in freier Natur und 4800 Kinder machen an jeder «Bad RagARTz» an spannenden Workshops ihre ersten spielerischen Erfahrungen mit der Kunstwelt, während im Jahre 2018 Sehende die Erfahrung machen konnten, wie es sich anfühlt, Kunst nicht zu sehen, sondern nur zu ertasten.

\section{Soziales Miteinander und Sensibilisierung der Jugend}

Wir spüren also: Die Kunst und die Auseinandersetzung mit Kunst birgt wesentlich mehr als nur den reinen Genuss in sich. Sie ist vielmehr ein wichtiger Lebensnerv der Gesellschaft. Sie lockt uns hinaus in die Natur, bewegt Geist und Körper. Sie fördert das für uns alle so wichtige soziale Miteinander, sie schärft den Blick für die Welt und das Gegenüber und sensibilisiert die Jugend mit einzigartigen Erfahrungen für die Schönheiten und die Herausforderungen ihrer Lebenswelt. Kaum verwunderlich also, ist die Auseinandersetzung mit der Kunst inzwischen auch eine viel beachtete Therapieform geworden, wenn es um die seelische Gesundheit des Menschen geht. In jedem

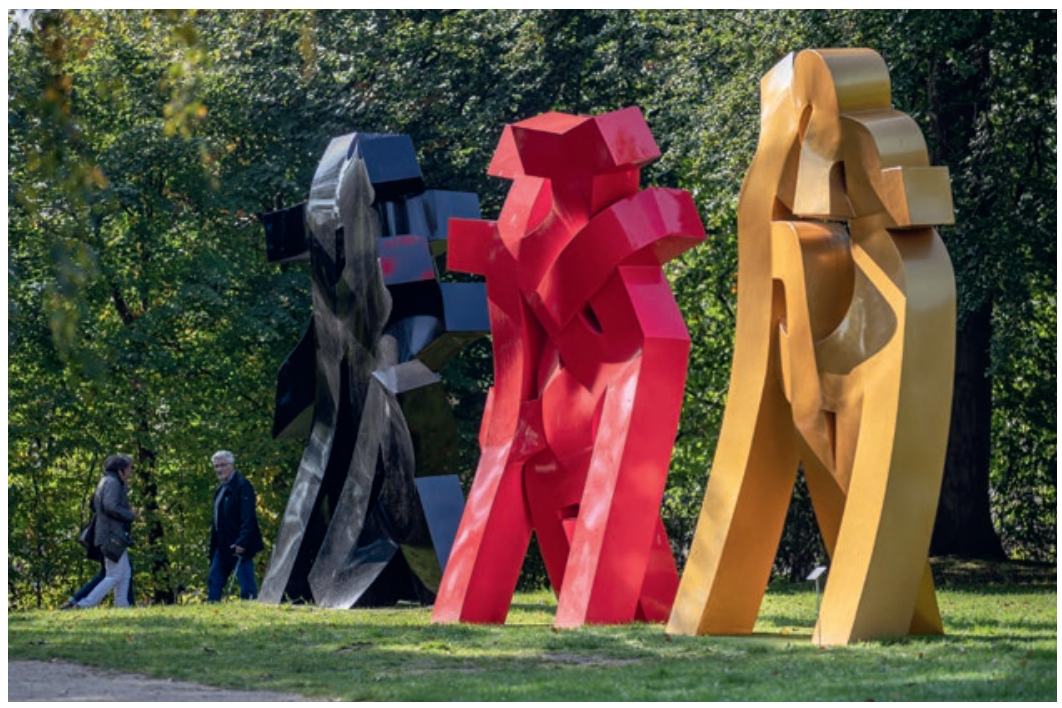

Liu Yonggang, Standing Character-Embrace of Love.

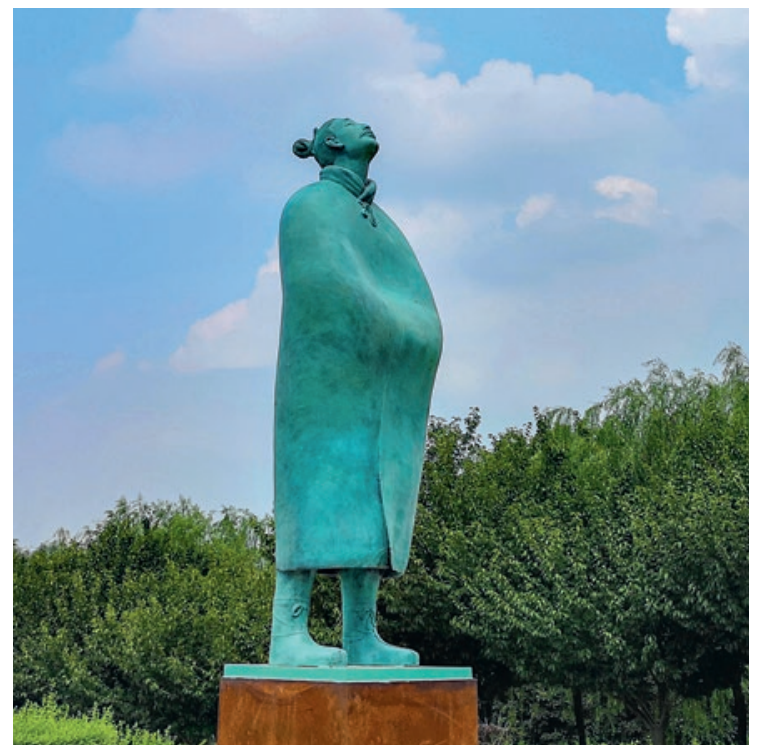

Lui Ruowang: Wenji Yang, Asking up, 2011.

Fall: Die Vielfalt der Kunst weckt die Lebensgeister und vermag zu Genuss, Entspannung und vielleicht sogar zu Glück zu verhelfen.

\section{Hervorragende Hotellerie und faszinierende Schluchtenwege}

Der ein- oder mehrtägige Ausflug nach Bad Ragaz lohnt sich aber auch weit über den Kunstgenuss hinaus: Die Kurgemeinde bietet nebst hervorragender Hotellerie eine vielfältige Gastronomie auf hohem Niveau sowie einen grosszügigen 18-Loch-Golfplatz mit wundervollem Naturerlebnis und grandiosem Blick auf die Berge der Bündner Herrschaft und des St.Galler Rheintales. Auch die Liebhaberinnen und Liebhaber erlesener Weine kommen mit Sicherheit auf ihre Kosten. Denn in unmittelbarer Nachbarschaft von Bad Ragaz befinden sich die berühmten Weingüter der Bündner Herrschaft sowie die idyllisch in den Rebbergen gelegenen Torkel. Empfehlenswert ist auch der Besuch der beeindruckenden Taminaschlucht, an deren Ende im alten Bad Pfäfers, dem Ursprungsort der Ragazer Heilkunst, im Rahmen der Triennale eine umfangreiche Kleinskulpturenausstellung zu sehen ist. In Valens wiederum ist in diesem Sommer auf dem Klinikareal eine Sonderausstellung mit beeindruckenden Skulpturen des 2019 verstorbenen Holzbildhauers Manfred Martin zu sehen.

Nähere Informationen:

www.badragartz.ch

\section{Bildnachweis}

Foto 1: FotoFetzer

Foto 2: NordArt Foto Wohlfromm

Foto 3: Nordart, Foto Wohlfromm 